\title{
A utilização dos anestésicos locais em odontologia: revisão de literatura
}

\author{
The use of local Anesthetics in Dentistry: Literature Review \\ El uso de Anestésicos locales en odontologia: Revisión Bibliográfica
}

Sabrina Ketulen do Nascimento Coelho ${ }^{1 *}$, Yuri da Silva Pimenta ${ }^{2}$, Jackeline Moraes de Andrade ${ }^{1}$, Núbia Afonso Silva ${ }^{1}$.

\section{RESUMO}

Objetivo: Analisar os estudos referentes as utilizações dos anestésicos locais, enfatizando o uso das soluções em pacientes sistemicamente comprometidos e as reações adversas que podem ocorrer. Revisão Bibliográfica: A descoberta das substâncias que provocam a perda da sensibilidade, causaram um marco para a medicina e através disto possibilitou as evoluções dos anestésicos locais que mais tarde seriam utilizadas nas clínicas médicas odontológicas. A escolha de um anestésico local é definida pelas características sistêmicas do paciente ou complicações que tenha adquirido no decorrer de sua vida. $O$ profissional deve levar em consideração o tempo do procedimento, a toxicidade que o anestésico pode ter e seu nível de concentração. As reações sistêmicas adversas aos anestésicos locais são divididas em três categorias: tóxica, psicogênica e alérgica, de acordo com o estudo feito, as reações alérgicas por esses anestésicos locais podem ocorrer, e a falta de domínio do cirurgião dentista sobre o assunto pode provocar uma complicação ao paciente. Considerações Finais: É importante que o cirurgião dentista busque o aprendizado contínuo, a fim de ter a melhor conduta durante atendimento odontológico.

Palavras-chave: Anestésicos locais, Pacientes, Reações adversas.

\section{ABSTRACT}

Objective: Analyze studies regarding the use of local anesthetics, emphasizing the use of solutions in systemically compromised patients and the adverse reactions that may happen. Bibliographic Review: The discovery of substances that cause loss of sensitivity, caused a milestone for medicine and through this enabled the evolution of local anesthetics that would later be used in dental clinics. The choice of a local anesthetic is defined by the patient's systemic characteristics or complications that he has acquired during his life. The professional must take into account the time of the procedure, the toxicity that the anesthetic may have and its level of concentration. Systemic adverse reactions to local anesthetics are divided into three categories: toxic, psychogenic and allergic, according to the study done, allergic reactions by these local anesthetics can occur, and the dentist's lack of mastery over the subject can cause a complication to the patient. Final Considerations: It is important that the dental surgeon seeks continuous learning in order to have the best conduct during dental care.

Keywords: Local anesthetics, Patients, Adverse reactions.

\section{RESUMEN}

Objetivo: Analizar estudios sobre el uso de anestésicos locales, enfatizando el uso de soluciones en pacientes sistémicamente comprometidos y las reacciones adversas que pueden ocurrir. Revisión Bibliográfica: El descubrimiento de sustancias que provocan pérdida de sensibilidad, supuso un hito para la medicina y, a través de ello, posibilitó la evolución de los anestésicos locales que luego serían utilizados en las clínicas dentales. La elección de un anestésico local se define por las características sistémicas del paciente o por las complicaciones que haya adquirido durante su vida. El profesional debe tener en cuenta el

${ }^{1}$ Centro Universitário do Norte (UNINORTE), Manaus - AM. *E-mail: Ketulen.nascimento@gmail.com

2 Universidade Federal do Amazonas (UFAM), Manaus - AM. 
tiempo del procedimiento, la toxicidad que pueda tener el anestésico y su nivel de concentración. Las reacciones adversas sistémicas a los anestésicos locales se dividen en tres categorías: tóxicas, psicógenas y alérgicas, según el estudio realizado, pueden producirse reacciones alérgicas por estos anestésicos locales, y la falta de dominio del dentista sobre el tema puede provocar una complicación para el paciente. Consideraciones Finales: Es importante que el cirujano dental busque un aprendizaje continuo para tener la mejor conducta durante el cuidado dental.

Palabras clave: Anestésicos locales, Pacientes, Reacciones adversas.

\section{INTRODUÇÃO}

Antes do surgimento do anestésico, a cirurgia era um procedimento extremamente doloroso para os pacientes, mas, em meados de 1842, o médico Crawford Williamson Long, utilizou, pela primeira vez o vapor de Éter sulfúrico com finalidade de anestesia geral (REZENDE JM, 2009). De acordo com Reis JA (2006) a notícia de operar sem dor utilizando o Éter sulfúrico foi publicada apenas em 1846, pelo odontologista William Thomas Green Morton e em 1847 por Horace Wells ao descobrir a anestesia geral com óxido nitroso.

Mas, somente em 1884 o anestésico local foi descoberto pelo médico Karl Koller; ao manusear experiências com a cocaína. Desde então, estudos foram realizados para que hoje pudéssemos utilizar anestésicos locais seguros, indispensáveis e eficazes na odontologia (REIS JA, 2009; TOBE M e SAITO S, 2015).

De acordo com Carvalho B, et al. (2013) e Pontanegra R, et al. (2017), afirmam que está disponível no mercado, uma série de anestésicos locais divididos em dois grandes grupos: os do tipo amida e os do tipo éster. Silva L, et al. (2019) discorre que essa variedade ocasiona dúvidas nos cirurgiões dentistas a respeito de qual sal anestésico e vasoconstritor utilizar em seus pacientes, isso faz com que muitos acabem padronizando apenas um tipo de solução para todos os procedimentos, o que consequentemente gera complicações, principalmente para pacientes sistemicamente comprometidos.

As complicações sistêmicas como urticária, rinite, angioedema, broncoespasmo, conjuntivite, distúrbio gastrointestinal, anafilaxia, acontecem imediatamente ou após a administração dos medicamentos e podem ser fatais para o paciente. Complicações das administrações locais associados aos anestésicos pode acarretar necrose tecidual e neurotoxicidade direta. A necrose ocorre devido à ruptura do tecido e está relacionada à natureza irritante da solução, a grandes volumes administrados ou a constrição da musculatura pelos vasoconstritores do anestésico local (AL), afirma (OUANOUNOU A, et al., 2020).

Portanto, é de suma importância que o futuro cirurgião dentista conheça a composição farmacológica, o mecanismo de ação, a indicação e a contraindicação de cada anestésico local, assim como as condições clínicas específicas do paciente, para evitar complicações irreparáveis ou interações medicamentosas (CARVALHO RWF, et al., 2010; PARISE GK, et al., 2017; SILVA L, et al., 2019).

À vista disso, o objetivo deste estudo será fazer uma revisão de literatura sobre a utilização dos anestésicos locais na odontologia e, com isso, enfatizar a história da evolução desses fármacos, a melhor indicação frente aos diferentes tipos de necessidades sistêmicas do paciente e as reações adversas causadas por eles.

\section{REVISÃO BIBLIOGRÁFICA}

\section{História dos Anestésicos locais}

A descoberta de substâncias que causam perda da sensibilidade foi um marco, sendo considerada uma das dez maiores descobertas da medicina (CARVALHO RWF, et al., 2010). De acordo com Tobe M, et al. (2018), após o surgimento da sedação com o éter sulfúrico e o óxido nitroso, em meados do século XIX, a anestesia local foi um evento inovador. Ele foi demonstrado pelo médico Karl Koller, em 1884, quando fez a administração da cocaína sob o globo ocular e descobriu, através dessa experiência, a sua ação causadora de insensibilidade (REIS JA, 2009). 
Conforme Grzanka A, et al. (2016), ao longo dos experimentos com a cocaína, percebeu-se que ocorriam efeitos colaterais, como: dependência, quando utilizada repetidamente, intoxicação sistêmica. Tobe $M$, et al. (2018) afirma que, a curta duração da ação anestésica da cocaína foi um problema no uso clínico. Ainda em 1884, o cirurgião Willian Halstead realizou o bloqueio do nervo alveolar inferior (NAl) e o fez, pela primeira vez, injetando a solução de cocaína com auxílio de uma seringa hipodérmica e uma agulha. Os resultados foram positivos quanto à analgesia, mas negativos no que se referem à duração (GIOVANNITTI JAJR, et al., 2013; FENCL JL, et al., 2015).

Em 1903, Heinrich Braun sugeriu a adição da adrenalina para atuar como torniquete "químico" para prolongar a duração anestésica (GIOVANNITTI JAJR, et al., 2013). Segundo Bulcão RP, et al. (2011) e Tobe M e Saito S (2015), em 1905, o bioquímico austríaco Alfred Einhorn, descobriu um substituto sintético da cocaína, e a denominou novocaína; ele concluiu que era quimicamente aceitável e possuía menor toxicidade.

De acordo com Tobe M, et al. (2018) e Waldlund DL, et al. (2017), a novocaína liderou o comércio local dos narcóticos, até o surgimento do primeiro anestésico do tipo aminoamida, denominada lidocaína. Esta, desenvolvida pelo químico sueco Nils Löfgren e pelo Bengt Lundquist, em 1943, era derivada da xilidina e possuía diferenças químicas da novocaína. Foi comercializada a partir de 1948 e recebida positivamente no mercado, tornando-se padrão para comparação com outros anestésicos locais, uma vez que tinha maior duração dessensibilizante e boa aceitação pelo organismo da maioria dos pacientes.

A descoberta da lidocaína foi essencial para o desenvolvimento dos anestésicos locais modernos, através dela outros da classe das amidas foram elaborados, como por exemplo, a mepivacaína sintetizada pelo químico sueco Ekenstam e introduzida no mercado em 1960. A bupivacaína, também sintetizada em 1957 por Ekenstam, só foi comercializada em 1963. Por sua vez, a prilocaína, idealizada por Nils Lofgren e Claes Tegner, foi divulgada apenas em 1959, já a articaína surgiu em meados de 1960 e divulgada apenas no final dos anos 70. Todos esses anestésicos mencionados possuem características farmacológicas semelhantes, no entanto são diferenciados, principalmente no que referem a tempo de ação e nível de toxicidade (LIMA RLFX, et al., 2010; WALDLUND DL, et al., 2017; TOBE M, et al., 2018).

\section{Utilização dos anestésicos locais}

De acordo com a pesquisa feita por Giovannitti JAJR, et al. (2013), a escolha de um anestésico local é definida pelas características sistêmicas do paciente, como peso, idade e possíveis complicações que 0 paciente tenha adquirido no decorrer de sua vida. Do ponto de vista clínico, o profissional deve levar em consideração o tempo do procedimento, a toxicidade que o anestésico pode ter e seu nível de concentração. $\mathrm{Na}$ odontologia, utilizam-se cinco soluções anestésicas disponíveis para realização de procedimentos. A principal solução utilizada nos Estados Unidos, que se tornou padrão-ouro, foi a lidocaína, que é apresentada em forma de cartucho na concentração de $2 \%$, porém é pouco utilizada devido à sua curta duração. A lidocaína 2\% combinada com epinefrina 1: 100:000 oferece maior duração, aproximadamente 60 minutos em anestesias pulpares e cerca de três a cinco horas em tecidos moles. A mepivacaína é semelhante à lidocaína, pois obtém uma excelente eficácia no seu início e duração. É fornecida em concentração de $2 \%$ com levonefrina 1: 20:000 e como solução pura de 3\%. Em procedimentos odontológicos, a mepivacaína de 3\% é a mais utilizada por apresentar propriedades vasodilatadoras mais fracas, possibilitando o uso para procedimentos de curta duração em pacientes que o uso de vasoconstritor não está indicado. A prilocaína tem uma menor potência que a lidocaína e se apresenta na concentração de $4 \%$ e $3 \%$. Contudo, é mais utilizada na concentração de $3 \%$. Por sua vez, a articaína é empregada na concentração de $4 \%$ contendo adrenalina 1: 100:000 ou 1: 200:000 e é utilizada em técnicas de infiltração e bloqueio na mandíbula, garantindo dessa forma, maior eficácia. Por fim, a bupivacaína, considerada o anestésico mais potente, é usada na forma de concentração de 0,5\% com epinefrina 1:100:000, seu uso é eficaz em procedimentos que requerem maior duração, como em cirurgias orais, possibilitando oito horas de bloqueio nervoso e reduzindo a dor no pós-operatório.

\section{Pacientes portadores de doenças cardiovasculares}

Num estudo feito na clínica odontológica no reino da Arábia Saudita por Abu-Mostafa N, et al. (2015), em que o objetivo era avaliar alterações hemodinâmicas e a frequência cardíaca, foram utilizadas três soluções 
de anestésicos diferentes: a lidocaína 2\% com epinefrina 1: 80:100, prilocaína 3\% com felipressina 0,03UI/ml e a mepivacaína $3 \%$ sem vasoconstritor. Participaram 45 pacientes hipertensos que seriam submetidos à extração de um dente, todos os pacientes possuíam a pressão arterial $\leq 160 / 100$, e os que possuíam a pressão sistólica $\geq 160$ e pressão diastólica $\geq 100$ foram excluídos; também foram excluídos pacientes que não utilizavam felipressina e epinefrina, pacientes grávidas e pacientes com alergia ao material anestésico utilizado no estudo. A pesquisa constatou que os pacientes que utilizaram lidocaína $2 \%$ com epinefrina 1 : $80: 100$ e prilocaína $3 \%$ com felipressina $0,03 \mathrm{Ul} / \mathrm{ml}$, no início do procedimento, apresentaram pressão arterial diminuída após a injeção e aumentada após a extração; porém quanto aos pacientes que utilizaram mepivacaína 3\% sem vasoconstritor, no início da injeção a pressão arterial aumentou, e após a extração baixou. $O$ resultado da pesquisa mostrou que os anestésicos lidocaína 2\% com epinefrina 1: 80:100 e prilocaína $3 \%$ com felipressina $0.03 \mathrm{Ul} / \mathrm{ml}$ são ideais para a utilização em pacientes hipertensos controlados, com pressão arterial $\leq 160 / 100$. Para pacientes que não estão devidamente controlados e precisaram passar por um procedimento de urgência odontológica, de acordo com Carvalho RWF, et al. (2010), o anestésico mais indicado será a mepivacaína $3 \%$, sem vasoconstritor ou a prilocaína $3 \%$ com vasoconstritor felipressina $0,03 \mathrm{Ul} / \mathrm{ml}$.

\section{Pacientes grávidas}

De acordo com Donaldson M e Goodchild JH (2012), os anestésicos locais usados na odontologia podem atravessar a barreira placentária e expor o embrião a desenvolver efeitos teratogênicos. Ele classificou a lidocaína com epinefrina ou prilocaína com felipressina como anestésicos de escolha pelo fato de serem de classificação B na gravidez pela $A$ Food and Drug Administration (FDA). Porém o autor destacou uma revisão de relatos de casos que fora publicada por Guay J (2009), relacionada à metemoglobinemia ocasionada pelos anestésicos locais e destacou que, de 79 casos, 12\% foram causados pela prilocaína.

Contudo, recomendou-se que a utilização da prilocaína seja evitada em mulheres grávidas, tendo em vista que sua molécula tem capacidade de atravessar rapidamente a placenta podendo levar à metemoglobinemia no feto e a contrações uterinas na gestante. Com isso, a lidocaína $2 \%$ com vasoconstritor adrenalina 1:100:000 é considerada segura para utilização em procedimentos realizados em mulheres grávidas que estão preferencialmente no segundo trimestre de gestação, afirma (LEE JM e SHIN TJ, 2017).

\section{Pacientes infantis}

Foi realizada uma revisão sistemática e uma metanálise por Tong HJ, et al. (2018), cujo objetivo era a procura da eficácia dos anestésicos articaína e lidocaína, que são utilizados nos procedimentos odontológicos em crianças. Os resultados mostraram que, na maioria dos procedimentos odontológicos realizados em crianças, são utilizadas a articaína $4 \%$ e a lidocaína $2 \%$, pois ambas oferecem o resultado desejado. 0 anestésico local articaína demonstrou ser eficaz na realização de procedimentos em crianças, tendo a mesma eficiência que a lidocaína.

Contudo, de acordo com Arrow PA (2012), foram atribuídas à articaína lesões pós-tratamento, como a mordedura de lábios e bochechas. Por se tratar de um anestésico com grande potencial de duração, é contraindicado para crianças com menos de quatro anos de idade. Para pacientes infantis que estiverem contraindicadas soluções anestésicas contendo epinefrina, Elbay ÜS, et al. (2016) relata que mepivacaína $3 \%$ sem vasoconstritor poderá ser empregada, pois possibilita uma analgesia semelhante à da lidocaína.

\section{Pacientes alérgicos e asmáticos}

De acordo com Malamed SF e Orr DL (2015), pacientes asmáticos podem ser intolerantes aos agentes sulfitastes como os metabissulfito de sódio, que são adicionados aos sais anestésicos como forma de antioxidantes. $O$ autor descreve que esses anestésicos que contêm epinefrina, levonefrina e bissulfito em sua composição, se forem administrados em pacientes asmáticos e alérgicos que são sensíveis a essas soluções, podem desencadear crises durante o procedimento odontológico.

Contudo, se o anestésico local contendo epinefrina for administrado com segurança, respeitando o volume e concentração, as crises asmáticas e alérgicas podem ser relativas. Ademais, se as soluções contendo em sua composição bissulfito de sódio forem contraindicadas, os anestésicos de escolha serão a prilocaína 3\% 
com o vasoconstritor felipressina $0,03 \mathrm{Ul} / \mathrm{ml}$ ou mepivacaína $3 \%$ sem vasoconstritor, pois eles demonstram ser eficazes para a realização do procedimento.

\section{Pacientes diabéticos}

Uma pesquisa realizada por Haji IU, et al. (2012), no Departamento de Cirurgia Oral e Maxilofacial da Faculdade e Hospital de Yenepoya Dental, na Índia, envolveu 50 pessoas diabéticas. Todas elas, que tomavam regularmente seu medicamento para o controle da doença e tinham seu nível glicêmico em jejum de $110-120 \mathrm{mg} / \mathrm{dL}$, foram submetidas à extração dentária. Os pacientes foram divididos em dois grupos de 25 pessoas, o grupo 1 recebeu a injeção com o anestésico local lidocaína $2 \%$, sem adrenalina, e o grupo 2 recebeu a injeção com o anestésico local lidocaína 2\%, com adrenalina 1: 80.000 . Foram avaliados os níveis glicêmicos antes da extração dentária e após os resultados, concluindo-se que em ambos os grupos não houve aumento significativo de glicemia. Foi levado em consideração que todos os pacientes submetidos ao procedimento faziam uso de medicamentos para o controle do nível de insulina do seu organismo e que isso possibilitou a realização da pesquisa.

Contudo, pacientes que não estejam fazendo o uso correto de medicamentos para o controle de sua glicemia, serão considerados pacientes descontrolados e, de acordo com Pinheiro AC, et al. (2015), o anestésico mais indicado será mepivacaína $3 \%$, sem vasoconstritor ou prilocaína $3 \%$, com vasoconstritor felipressina $0,03 \% \mathrm{UI} / \mathrm{ml}$.

\section{Pacientes com problema endócrino}

Indivíduos que possuem dificuldade na produção de hormônio triiodonteranina T3 e tiroxina T4 são considerados pacientes com problema endócrino relacionado diretamente à glândula tireoide. Esse problema afeta diretamente o crescimento e desenvolvimento desses pacientes. Entretanto, pessoas que apresentam uma superprodução desses hormônios estão sujeitas a complicações graves que podem ocasionar risco de vida, descreve (PINTO A e GLICK M, 2002).

Malamed SF e Orr DL (2015) demonstra que pacientes que têm este distúrbio endócrino, mas fazem uso do medicamento indicado para controlar seu nível de hormônio, podem realizar procedimentos normalmente, contudo o profissional deverá tomar precauções em utilizar vasoconstritor, contendo epinefrina, devendo empregar a menor concentração de epinefrina, tal como em concentrações de 1: 200:000 - é preferível 1: 100.000 ou 1: 50.000 . Entretanto, para pacientes que não estão realizando o devido tratamento para a regularização dos níveis de hormônio, a epinefrina estará contraindicada, pois acarretará o aumento de adrenalina no paciente, levando-o a complicações.

\section{Distinções entre as reações adversas provocadas pelos anestésicos locais do tipo amida na odontologia.}

Existem diferentes tipos de reações provocadas pelos anestésicos locais e Henderson S (2011) afirmava que, as reações sistêmicas adversas aos anestésicos locais podem ser divididas em três categorias: tóxica, psicogênica e alérgica.

\section{Toxicidade}

O estudo aponta que, em virtude da superdosagem, os pacientes podem apresentar sintomas, desde leves até mais graves que, em alguns casos, podem levar o paciente à morte, conforme o fármaco vá se espalhando pelo sistema circulatório. Em concentrações mais altas, convulsões ocorrem, sendo a consequência inicial com risco de morte, devido à overdose de anestésico local. Presumivelmente, isso se deve à depressão seletiva dos tratos inibitórios corticais, permitindo a atividade sem oposição das vias excitatórias. Essa seletividade é perdida, à medida que as concentrações séricas aumentam ainda mais, e todas as vias são inibidas, resultando em coma, parada respiratória e, eventualmente, colapso cardiovascular (BECKER DE, 2014).

De acordo com Henderson S (2011), os efeitos colaterais tóxicos são predominantemente neurológicos e incluem excitabilidade ou agitação, sedação, tontura, fala arrastada, alteração de humor, diplopia, desorientação e contração muscular. 


\section{Psicogênese}

Henderson S (2011) afirmava que a síncope é o sintoma mais comum causado pela ansiedade e medo. Outros sintomas incluem ataques de pânico, hiperventilação, náusea, vômito e alterações na frequência cardíaca ou pressão arterial, que podem causar palidez. Há uma alta proporção de reações psicossomáticas à anestesia local em uma consulta odontológica, que muitos especialistas consideram erroneamente uma reação adversa à anestesia e alergias em particular (KUVIN AV, et al., 2018).

\section{Reações alérgicas}

Consoante o estudo, o sistema imunológico reage através de dois mediadores às reações alérgicas dos anestésicos locais na odontologia, são eles: Tipo I, que se entende como reação sérica anafilática; e tipo IV, reação mediada por células. Esta é uma resposta imediata enquanto aquela é uma resposta tardia. Os níveis séricos de lgE (Tipo 1; Reação Anafilática) diminuem em uma hora, após o início de uma reação alérgica. Em seguida à diminuição inicial, há um aumento acentuado na concentração plasmática de anticorpos lgE que permanece elevada por 72 horas, após uma resposta adversa. O sistema complemento também pode mediar uma resposta alérgica retardada com anestesia local (tipo IV; reação mediada por células) (SPECA SJ, et al., 2010).

Em concordância com o estudo, sintomas da alergia tipo I tendem a ocorrer alguns minutos após a injeção: os lábios e as áreas periorbitais incham (angioedema); o paciente pode ficar agitado e há urticária e prurido generalizados e diarreia; aperto do peito, com sibilos e dificuldade em respirar, pode ocorrer; há possibilidade de haver uma queda na pressão sanguínea e um pulso rápido, podendo ser acompanhado por rubor da pele ou erupção cutânea. Já o tipo IV é um atraso da reação de hipersensibilidade, mediada por linfócitos sensibilizados, com características típicas da seguinte forma: podem se manifestar de várias maneiras, sendo a síncope a mais comum; outros sintomas incluem ataques de pânico, hiperventilação, náusea, vômito e alterações na frequência cardíaca ou pressão arterial, que podem causar palidez. Tal como ocorre com o grupo psicogenético, esses sintomas podem ser diagnosticados, erroneamente, como reações alérgicas e também podem imitar os sinais de alergias como rubor da pele, erupção cutânea vermelha manchada, edema e broncoespasmo (HENDERSON S, 2011)

De acordo com Arrow PA (2012), foram atribuídas à articaína lesões pós-tratamento, como a mordedura de lábios e bochechas. Por se tratar de um anestésico com grande potencial de duração, é contraindicado para crianças com menos de quatro anos de idade. Entretanto, se ao paciente infantil estiverem contraindicadas soluções anestésicas contendo epinefrina, Elbay ÜS, et al. (2016) relata que mepivacaína $3 \%$ sem vasoconstritor poderá ser empregada, pois possibilita uma analgesia semelhante à da lidocaína.

O tempo da anestesia local nos tecidos moles é maior do que a anestesia pulpar, e com isso lesões induzidas de tecidos moles podem ocorrer, descreve Bagattoni S (2020), onde realizou uma pesquisa a respeito das lesões de tecidos moles em crianças e o anestésico local relacionado. A pesquisa chegou à conclusão de que independente do sal anestésico empregado, os monitoramentos dos pais e a dose de administração pelos profissionais foram de suma importante para que não ocorressem as lesões teciduais.

De acordo com Henderson S (2011), o paciente deve ser encaminhado para uma investigação mais aprofundada para confirmar se o anestésico local ou outro possível alérgeno (por exemplo, excipiente, látex) foi a causa dos efeitos adversos.

A investigação de pacientes com suspeita de alergia a $A L$ deve começar com uma história detalhada que deve determinar se serão necessários teste e desafio cutâneos (BHOLE MV, et al., 2012). O processo investigativo que elimina outras possíveis reações e alérgenos é discutido até a identificação e teste de alternativas apropriadas de tolerância, para concluir com opções alternativas de anestésico local que são desafiadas pela segurança clínica (ALLEN G, et al., 2017).

Pacientes com verdadeira alergia a anestésicos locais amida apresentam um desafio ao dentista em fornecer cuidados adequados com o manejo adequado da dor intraoperatória (DE PASQUALE MTA, et al., 2018). Segundo Kuvin AV, et al. (2018), os seguintes dados do histórico do paciente são considerados de alto risco para o desenvolvimento de uma reação alérgica: uma reação alérgica a medicamentos já 
experienciada, incluindo casos de paciente com risco de vida e hospitalização; alergia a vários anestésicos (alergia cruzada); falta de opinião escrita de um alergista-imunologista sobre o estado de saúde e a tolerância aos anestésicos locais, além de dados de exames laboratoriais incompletos ou desatualizados. Um baixo risco de desenvolver uma reação alérgica inclui anamnese na qual não haja confirmação de alergia a medicamentos.

Explicações mais comuns para as sequelas sistêmicas aos anestésicos locais estão relacionadas à overdose de drogas, absorção rápida, injeção intravascular, cardiovascular, sistema nervoso central e a reações psicogênicas ou idiossincráticas. Apesar de ser relatada com frequência pelos pacientes, uma verdadeira reação alérgica ao anestésico local é rara, com relatos de que uma alergia dessa natureza é responsável por apenas $1 \%$ das reações sistêmicas adversas (ALLEN G, et al., 2017).

A prevalência de reações alérgicas aos anestésicos locais, de acordo com as estatísticas mundiais, é extremamente baixa. Entre todas as complicações da anestesia local, sua proporção é de $0,1 \%$ a $0,5 \%$. Todos os anos, casos únicos de reações alérgicas aos anestésicos locais de todos os grupos químicos são registrados no mundo. Há uma alta proporção de reações psicossomáticas à anestesia local em uma consulta odontológica, que muitos especialistas consideram erroneamente uma reação adversa à anestesia e alergias em particular (KUVIN AV, et al., 2018).

\section{CONSIDERAÇÕES FINAIS}

Dessa forma, entende-se que são utilizados anestésicos locais específicos para pacientes acometidos por doenças sistêmicas. Também se esclarece que a prática de uma anamnese completa é imprescindível. Isso porque ela serve de suporte à compreensão sobre os sinais e sintomas que o paciente pode apresentar, seja antes ou após o uso da anestesia local. Por fim, os resultados das pesquisas evidenciam a importância da busca continuada dos cirurgiões-dentistas pelas atualizações dos conhecimentos neste assunto. Sabendo que o uso indiscriminado de drogas pode gerar reações adversas no organismo, é de suma importância que o profissional saiba as indicações e contraindicações desses fármacos.

\section{REFERÊNCIAS}

1. ABU-MOSTAFA N, et al. Um estudo clínico prospectivo randomizado comparou o efeito de vários tipos de cartuchos de anestésicos locais em pacientes hipertensos durante a extração dentária. J. Clin. Exp. Dent., Madrid, 2015; 7(1): 84-88.

2. ALLEN G, et al. Case Report: Investigation and Diagnosis of na Immediate Allergy to Amide Local Anesthetic in a Pediatric Dental Patient. Australian Dental Journal.Richmond, 2017; 62(2): 241-245.

3. ARROW PA. Comparison of articaine $4 \%$ and lignocaine $2 \%$ in block and infiltration analgesia in children. Revista Australian Dental Journa, Western Australia, 2012; 57(3): 325-333.

4. BAGATTONI S. Lesões de tecidos moles auto-induzidas após anestesia dentária em crianças com e sem deficiência intelectual. Um estudo prospectivo. J. Paediatr Dent, Bolonha, 2020; 21 (5): 617-622

5. BECKER DE. Adverse Drug Reactions in Dental Practice. The American Dental Society Of Anesthesiology. Chicago, 2014; 61(1), 26-34.

6. BHOLE MV, et al. IgE-mediated allergy to local anesthetics: separating fact from perception: a UK perspective. British Journal of Anesthesia, 2012; 108(6), 903-911.

7. BINA B, et al. True allergy to amide local anesthesics: A review and presentation. Anesth Prog., Brokelyn, 2018; 65(2), 119-123.

8. BULCÃO RP, et al. Procaína: efeitos farmacológicos e toxicológicos. Revista De Ciências Farmacêutica Básica e Aplicada, Araraquara, 2011; 32(3), 297-303.

9. CARVALHO B, et al. O emprego dos anestésicos locais em Odontologia: Revisão de Literatura. Rev. Bras. Odontol. Rio de Janeiro, 2013; 70(2), 178-181.

10. CARVALHO, R. W. F. et al. Anestésicos Locais: Como Escolher e Prevenir Complicações Sistémicas. Revista Portuguesa de Estomatologia, Medicina Dentária e Cirurgia Maxilofacial, Lisboa, 2010; 51 (2), 113 -119.

11. DE PASQUALE MTA, et al. Delayed-type allergy to articaine with cross-reactivity to other local anesthetics from the amide group. American. Academy of Allergy, Asthma \& Immunology. Milwaukee, 2018; 6(1), 305-306.

12. DONALDSON M, GOODCHILD JH. Gravidez, aleitamento materno e medicamentos utilizados em odontologia. Revista J. American Dental Association, Filadélfia, 2012; 143(8), 858-871.

13. ELBAY ÜŞ, et al. Efeitos de duas soluções anestésicas diferentes na dor da injeção, eficácia e duração da anestesia de tecidos moles com bloqueio do nervo alveolar inferior para molares primários. Revista Journal Of Clinical Pediatric Dentistry, Yuvacık Turquia, 2016; 40(3), 456-463. 
14. FENCL JL, et al. Local Anesthetic Systemic Tooxicity Perioperative Implications. AORN JOURNAL, Califórnia, 2015; 101(6), 697-700.

15. GIOVANNITTI JR, et al. Farmacologia dos anestésicos locais utilizados em cirurgia oral: 3. Ed. Cincinnati: Editora Elsevier, Califórnia, 2013; 453-465p.

16. GRZANKA A, et al. Hypersensitivity lo local anesthetics. Anaesthesiol Intensive Ther, Poznan, 2016; 48(2), $128-134$.

17. GUAY J. Metemoglobinemia relacionado a anestésicos locais: um resumo de 242 episódios J. anesthesia \& analgesia Estados Unidos, 2009; 108(3), 837-845.

18. HAJI IU, et al. Estudo sobre a concentração de glicose no sangue em pacientes com diabetes submetidos à extração dentária sob anestesia local com e sem adrenalina. Revista J Basic Clin Physiol Pharmacol, Délhi, 2012; 23(4), 169171.

19. HENDERSON S. Allergy to Local Anesthetic Agents used in Dentistry - What are the Signs, Symptoms, Alternative Diagnoses and Management Options. Dent Update, Guilford, 2011; 38(6), 410-412.

20. KUVIN AV, et al. Dental care for patients at risk of adverse reaction to local anesthetics. Central de Pesquisa de Odontologia e Cirurgia Maxilofacial. Moscou, 2018; 97(6), 63-66.

21. LEE JM, SHIN TJ. Uso de anestésicos locais para tratamento odontológico durante a gravidez; segurança para parturiente. J. Dent Anesth Pain Med., Seul, 2017; 17(2), 81-90.

22. LIMA RLFX, et al. Análise comparativa entre articaína e mepivacaína na anestesia da região palatina: estudo-piloto. Rev. Cir. Traumatol. Buco-maxilo-fac., Camaragibe, 2010; 10(4), 111-116.

23. OUANOUNOU A, et al. Adverse drug reactions in dentistry. International Dental Journal, Toronto, 2020; 70(2), 79-84.

24. PARISE GK, et al. Sais anestésicos utilizados na odontologia: revisão de literatura. Journal Of Oral Investigatins, Passo Fundo. 2017; 6(1), 75-84.

25. PINHEIRO AC, et al. Conhecimento dos dentistas sobre sinais e sintomas da toxicidade sistêmica de soluções anestésicas locais. Revista RGO, Rev. Gaúch. Odontol. Campinas, 2015; 63(1), 41-46.

26. PINTO A, GLICK M. Manejo de pacientes com doença da tireóide: considerações sobre saúde bucal. Revista J Am Dent Association, Filadélfia, 2002; 133(7), 849-858.

27. PONTANEGRA R, et al. Análise do conhecimento de graduandos em odontologia sobre o uso de anestésico local em pacientes com necessidades especiais. FOL/Unicamp, Linz, 2017; 27(1), 5-14.

28. REIS J, ALMIRO D. O primeiro a utilizar anestesia em cirurgia não foi um dentista. Foi o médico Crawford Williamson Long. Rev. Bras. Anestesiol, Campinas, 2006; 56(3), 304-324.

29. REIS J, ALMIRO D. Sigmund Freud (1856-1939) e Karl Köller (1857-1944) e a descoberta da anestesia local. Rev. Bras. Anestesiol, Campinas, 2009; 59(2), 244-257.

30. REZENDE JM. À Sombra do plátano: crônicas de história da medicina [online]. Editora Fap-Unifesp, São Paulo, 2009; 2(10), 103-108.

31. SILVA L, et al. Anestésicos locais em clínica universitária odontológica: conhecimento dos graduandos acerca de efeitos adversos e tóxicos. Revista Saúde (Sta. Maria), Quixadá, 2019; 45(2), 1-10.

32. SPECA SJ, et al. Allergic reactions to local anesthesic formulations. Derpatment of Anesthesiology, University of Pittsburgh School of Dental Medicine, Pittsburgh, Dent Clin North Am, Pitssburgh, 2010; 54(4), 655-644.

33. STANLEY F. MALAMED, DANIEL L. ORR. Asthma, 7. ed. Canadá: Editora Elsevier, 2015; 214-231p

34. TOBE M, SAITO S. History of T-cain: a local anesthetic developed and manufactured in japan. Journal Of Anesthesia, Gunma, 2015; 29(5), p. 763-768.

35. TOBE M, et al. The History and progress off local anestesia: multiple approaches to elongate the action. Journal Of Anesthesia, Gunma, 2018; 34(32), 13-17.

36. TONG HJ, et al. Eficácia anestésica da articaína versus lidocaína na odontologia infantil: uma revisão sistemática e meta-análise. Revista International Journal Of Pediatric Dentistry, Singapura, 2018; 28(4), 347-360.

37. WALDLUND DL, et al. Toxicidade sistêmica do anestésico local. AORN JOURNAL, Califórnia, 2017; $106(5), 367-377$. 